CLINICAL STUDY

\title{
Adiponectin and cardiac geometry and function in elderly: results from two community-based cohort studies
}

\author{
Stefan Gustafsson, Lars Lind ${ }^{1}$, Björn Zethelius ${ }^{2}$, Per Venge ${ }^{1}$, Allan Flyvbjerg ${ }^{3}$, Stefan Söderberg ${ }^{4}$ and Erik Ingelsson \\ Department of Medical Epidemiology and Biostatistics, Karolinska Institutet, PO Box 281, SE-171 77 Stockholm, Sweden, Departments of ${ }^{1}$ Medical \\ Sciences and ${ }^{2}$ Public Health and Caring Sciences, Uppsala University, Uppsala, Sweden, ${ }^{3}$ The Medical Research Laboratories, Clinical Institute, and Medical \\ Department M (Diabetes and Endocrinology), Aarhus University Hospital, Aarhus, Denmark and ${ }^{4}$ Department of Public Health and Clinical Medicine, \\ Cardiology, Umeå University Hospital, Umeå, Sweden
}

(Correspondence should be addressed to E Ingelsson; Email: erik.ingelsson@ki.se)

\begin{abstract}
Objective: Several smaller studies have indicated that adiponectin might be associated with left ventricular (LV) mass and function, but community-based studies with adequate sample size and adjustment for potential confounders are lacking. Our objective was to investigate such associations in two large community-based studies of elderly.

Design: Cross-sectional.

Methods: We evaluated cross-sectional relations between serum adiponectin and echocardiographic measures of cardiac geometry and function (LV mass index, LV relative wall thickness, LV end-diastolic diameter, left atrial diameter, ejection fraction, $\mathrm{LV}$ isovolumic relaxation time, and E/A ratio) in 954 70-year-old participants (50\% women) of the Prospective Investigation of the Vasculature in Uppsala Seniors (PIVUS), and in 427 71-year-old men from the Uppsala Longitudinal Study of Adult Men (ULSAM).

Results: In models adjusted for age, sex, body mass index, systolic blood pressure, antihypertensive treatment, antidiabetic treatment, lipid-lowering medication, fasting blood glucose, total cholesterol, high-density lipoprotein cholesterol, creatinine, and smoking, adiponectin was inversely associated with ejection fraction in men $(\beta,-1.62 ; 95 \%$ confidence interval $(\mathrm{CI}),-2.50,-0.75$ in PIVUS; $\beta,-1.35 ; 95 \% \mathrm{CI},-2.41,-0.29$ in ULSAM), but not in women. After additional adjustment for N-terminal pro-brain natriuretic peptide (NT-proBNP), the association between adiponectin and ejection fraction was attenuated $(\beta,-0.98 ; 95 \% \mathrm{CI},-1.86,-0.10$ in PIVUS; $\beta,-0.75 ; 95 \%$ CI, $-1.84,0.35$ in ULSAM).

Conclusions: Serum adiponectin concentrations were associated with ejection fraction in men, and these associations were partially attenuated by NT-proBNP. Our results imply that adiponectin may be associated with systolic function through pathways that involve natriuretic peptides.
\end{abstract}

European Journal of Endocrinology 162 543-550

\section{Introduction}

Cardiovascular disease (CVD) is a leading cause of death accounting for about one-third of the total number of deaths in the world. It is a major contributor to the increased burden on public health care $(1,2)$. Recent studies have demonstrated the critical role of adipose tissue as an endocrine organ that by secretion of adipocytokines can affect several processes that can lead to the development of CVD (3). Adiponectin, the most abundant adipocytokine, is recognized as a key regulator of insulin resistance (IR), tissue inflammation, and other pathophysiological processes (4).

Recently, the role of adiponectin in relation to cardiac geometry and function has attracted some attention. Several studies performed in small study samples, mostly in patients with preexisting disease, such as type 2 diabetes, hypertension, or obesity, have reported inverse associations between adiponectin and left ventricular (LV) mass (5-9), and diastolic dysfunction (7, 9). Similarly, a larger sample of healthy Japanese men has presented an association between lower levels of adiponectin and dichotomous electrocardiography (ECG)-LV hypertrophy (ECG-LVH) as an outcome (10). There are, however, very few large, community-based studies with adequate adjustment for potential confounders to elucidate these relations in greater detail; to our knowledge, only one prior study has investigated the relation of adiponectin with $\mathrm{LV}$ geometry and function in a moderately large sample of healthy individuals (11). In this study, circulating adiponectin increased in parallel with brain natriuretic peptide (BNP), and was positively associated with higher LV end-diastolic diameter (LVEDD) which could indicate depressed cardiac function. A potential role for the balance of adiponectin and natriuretic peptides in the development of cardiac 
dysfunction has also been indicated by studies showing increased levels of both adiponectin and N-terminal proBNP (NT-proBNP) in patients with heart failure (12-14).

Based on these previous data, we hypothesized that circulating adiponectin would be associated with LV geometry and function, and that this would be partially mediated through pathways involving natriuretic peptide levels. We tested these hypotheses in two large, community-based samples of the elderly who underwent echocardiography and measurements of adiponectin and NT-proBNP.

\section{Subjects and methods}

\section{Study samples}

The Prospective Investigation of the Vasculature in Uppsala Seniors (PIVUS) has been described in detail previously (15) and at http://www.medsci.uu.se/pivus/ pivus.htm. In brief, all 70-year-old individuals residing in Uppsala, Sweden, between April 2001 and June 2004 were eligible for the study. By random selection, 2025 subjects were invited, of which 1016 subjects $(50 \%$ women) were examined within 1 month of their 70th birthday in order to standardize for age. After exclusion of individuals without valid adiponectin measurements $(n=9)$, echocardiographic readings $(n=30)$, or all clinical characteristics needed for multivariable analysis $(n=23)$, the eligible study sample consisted of 954 participants.

The Uppsala Longitudinal Study of Adult Men (ULSAM) has been described elsewhere (16) and at http://www.pubcare.uu.se/ULSAM/. Briefly, the study was initiated in 1970-74 when all the 50-year-old men- living in Uppsala County, Sweden, were invited to participate. The participants were re-examined in August 1991 to May 1995 at the approximate age of 71 years. Of 1681 invited individuals, 1221 (73\% of those still alive and living in Uppsala) participated in the follow-up. An echocardiographic examination was performed in a random sub-sample of 482 individuals, and after exclusion of participants without valid adiponectin measurements $(n=16)$ or all clinical measures used in the analyses $(n=39)$, the eligible sample consisted of 427 individuals. Both studies were approved by the ethics committee of Uppsala University, and all participants provided written informed consent.

\section{Assessment and definitions of clinical covariates}

Similar and standardized methods were used in both studies for the collection of anthropometrical measurements, blood pressure, and fasting blood and urine samples, and included a questionnaire regarding medical history, smoking habits, and regular medication. All participants went through an overnight fast prior to the investigation, in which both medication and smoking were not allowed. Body mass index (BMI) was calculated by dividing weight $(\mathrm{kg})$ with squared height $\left(\mathrm{m}^{2}\right)$. Blood pressure was measured by a calibrated mercury sphygmomanometer to the nearest even $\mathrm{mmHg}$ after at least $30 \mathrm{~min}$ of rest, and the mean of three (PIVUS) or two (ULSAM) recordings was used. Lipid variables, creatinine, and fasting blood glucose were measured using standard laboratory techniques. NT-proBNP was measured in PIVUS and ULSAM with a sandwich immunoassay on an Elecsys 2010 (Roche Diagnostics) at an analytical range of 20-35 $000 \mathrm{ng} / \mathrm{l}$. The total coefficient of variation $(\mathrm{CV})$ was $3.3 \%$ at a level of $209 \mathrm{ng} / \mathrm{l}$ and was $3.0 \%$ at a level of $7.4 \mathrm{ng} / \mathrm{l}$.

Echocardiographic parameters were measured with a comprehensive two-dimensional and Doppler echocardiography, which was performed with an Acuson XP124 cardiac ultrasound unit (Acuson, Mountain View, CA, USA) $\sim 1$ week after the main examination by Dr Lind who was blinded to the clinical data (17). The echocardiographic measures included LVEDD, left atrial diameter, ejection fraction, $\mathrm{LV}$ isovolumic relaxation time, E/A ratio, interventricular septal thickness (IVST), and LV posterior wall thickness (PWT). LV relative wall thickness (RWT) was calculated as (IVST + PWT)/ LVEDD and LV mass $(\mathrm{LVM})$ as $0.8 \times(1.04 \times(\mathrm{IVST}$ + LVEDD + PWT $)^{3}-$ LVEDD $\left.^{3}\right)+0.6$ g (18). LVM index (LVMI) was obtained by indexing LVM divided by height to the power of $2.7\left(\mathrm{LVM} / \mathrm{m}^{2.7}\right)$ (19). Ejection fraction was calculated from M-mode recordings according to the method of Teichholz (20). LV geometry was categorized as: normal $\left(\mathrm{LVMI} \leq 51 \mathrm{~g} / \mathrm{m}^{2.7} ; \mathrm{RWT} \leq 0.45\right)$, eccentric $\mathrm{LVH}\left(\mathrm{LVMI}>51 \mathrm{~g} / \mathrm{m}^{2.7} ; \mathrm{RWT} \leq 0.45\right)$, concentric remodeling $\left(\mathrm{LVMI} \leq 51 \mathrm{~g} / \mathrm{m}^{2.7} ; \mathrm{RWT}>0.45\right)$, and concentric $\mathrm{LVH}\left(\mathrm{LVMI}>51 \mathrm{~g} / \mathrm{m}^{2.7}\right.$; RWT $\left.>0.45\right)(21)$.

\section{Adiponectin measurements}

Adiponectin in samples from PIVUS, frozen for 1-3 years, was analyzed with a double-antibody RIA (Linco Research, St Louis, MO, USA). The total CV for adiponectin was $15.2 \%$ at low $(2-4 \mu \mathrm{g} / \mathrm{ml})$ and $8.8 \%$ at high $(26-54 \mu \mathrm{g} / \mathrm{ml})$ levels. The measurement of adiponectin in ULSAM has been described in detail previously (22). In brief, serum adiponectin was measured in a validated in-house time-resolved immunofluorometric assay based on commercial reagents from R\&D Systems (Abingdon, UK). The analyses were done in samples frozen at $-70{ }^{\circ} \mathrm{C}$ for $11 \pm 2$ years, without previous thaw-freeze cycles, collected at examination at the age of 71 between 1991 and 1995. The average intra-assay and inter-assay CV averaged $<5$ and $10 \%$ respectively (12).

\section{Statistical methods}

All variables were normally distributed, except E/A ratio and fasting glucose; these two were logarithmically transformed in order to promote normality. 
As circulating adiponectin concentrations may differ between sexes, levels were $z$-score transformed in each sex separately before pooling data in PIVUS. This approach also allowed comparisons of $\beta$-coefficients in PIVUS and ULSAM; thus, the $\beta$-coefficients presented represent a 1-S.D. increase in adiponectin. The associations between adiponectin, cardiac geometry, and function were analyzed in age- and sex-adjusted analyses, as well as in multivariable linear regression models with echocardiographic measures (LVMI, relative wall thickness, end-diastolic diameter, left atrial diameter, ejection fraction, isovolumic relaxation time, and $\mathrm{E} / \mathrm{A}$ ratio) as dependent variables in separate models. The multivariable regression models were adjusted for age, sex (only in PIVUS), BMI, systolic blood pressure, antihypertensive treatment, antidiabetic treatment, lipid-lowering medication, log fasting blood glucose, total cholesterol, high-density lipoprotein (HDL) cholesterol, creatinine, and smoking (no/former/ current). To address a potential mediating role of natriuretic peptides in the relation of adiponectin with cardiac function, we added NT-proBNP to the multivariable-adjusted models with ejection fraction, isovolumic relaxation time, or E/A ratio as an outcome. Interactions were tested by two-way interaction terms between sex and adiponectin with echocardiographic variables as the dependent variables in separate models. Also, to avoid a potentially confounding effect of co-morbidities with heart disease, analyses were performed in sub-samples consisting of 804 (PIVUS) and 356 (ULSAM) individuals without a history of myocardial infarction, heart failure, angina pectoris or interventional treatment of the coronary arteries, or with significant valvular diseases detected on the echocardiogram. Adiponectin levels in relation to groups of cardiac geometry were calculated as ageand sex-adjusted least square means of adiponectin grouped by categories of cardiac geometry in the PIVUS study, and the results were visualized in a figure. Twotailed significance values were given with $P<0.05$ being regarded as significant. The statistical software package STATA 10.1 (Stata Corporation, College Station, TX, USA) was used for all analyses.

\section{Results}

Table 1 presents the clinical characteristics along with measures of cardiac geometry and function, separately for PIVUS and ULSAM.

\section{Associations between adiponectin and measures of cardiac geometry}

In PIVUS, adiponectin was inversely associated with LVMI, LV RWT, and left atrial diameter in age- and sexadjusted analyses (Table 2, left column). Consistent with this, adiponectin concentrations were significantly lower in individuals with eccentric hypertrophy, concentric remodeling, and concentric hypertrophy than in individuals with normal cardiac geometry (Fig. 1). The associations of adiponectin with LV geometry were nonevident in multivariable-adjusted analyses (Table 2, right column). In secondary analyses, associations between adiponectin and LVM (without indexing for height) were almost identical to those incorporating LVMI (data not shown). Exploratory analyses demonstrated that BMI was the most important confounder of the associations of adiponectin and cardiac geometry as the addition of BMI to the ageand sex-adjusted analyses rendered all associations nonsignificant $(P=0.52 ; 0.14$; and 0.91$)$. In ULSAM, adiponectin was not significantly associated with measures of cardiac geometry in either age- and sexadjusted or multivariable-adjusted analyses (Table 3).

\section{Associations of adiponectin and measures of cardiac function}

In multivariable-adjusted models including BMI, circulating adiponectin was inversely associated with ejection fraction in PIVUS (Table 2) as well as in ULSAM (Table 3). For each s.D. increase of adiponectin, ejection fraction decreased by $1.2 \%$ (PIVUS) or $1.3 \%$ (ULSAM). Sex was found to be an effect modifier in the association of adiponectin with ejection fraction $(P$ for

Table 1 Clinical characteristics and echocardiographic measures ${ }^{a}$.

\begin{tabular}{lll}
\hline & $\begin{array}{l}\text { PIVUS } \\
(n=954)\end{array}$ & $\begin{array}{l}\text { ULSAM } \\
(n=427)\end{array}$ \\
\hline Clinical characteristics & & \\
Age & $70.2(0.2)$ & $71.2(0.4)$ \\
Women (\%) & 50 & 0 \\
BMI (kg/m $\left.{ }^{2}\right)$ & $27.1(4.3)$ & $26.1(3.2)$ \\
Systolic blood pressure (mmHg) & $150(23)$ & $149(19)$ \\
Antihypertensive treatment (\%) & 31 & 37 \\
Total cholesterol (mmol/l) & $5.4(1.0)$ & $5.8(1.0)$ \\
HDL cholesterol (mmol/l) & $1.5(0.4)$ & $1.3(0.3)$ \\
Lipid-lowering treatment (\%) & 16 & 9 \\
Fasting plasma glucose (mmol/l) & $5.4(1.6)$ & $5.8(1.4)$ \\
Diabetes treatment (\%) & 6.6 & 5.4 \\
Creatinine ( $\mu$ mol/I) & $80(20)$ & $94(14)$ \\
Smoking (\%) & Never: 49 & Never: 40 \\
& Previous: 41 & Previous: 40 \\
& Current: 10 & Current: 20 \\
Adiponectin (mg/l) & $7.1(4.3)$ & $10.6(4.4)$ \\
Echocardiographic measures & & \\
LV mass index (g/m $\left.{ }^{2.7}\right)$ & $43.1(13.2)$ & $58.8(13.2)$ \\
LV relative wall thickness & $0.44(0.09)$ & $0.41(0.06)$ \\
LV end-diastolic diameter (mm) & $47(5)$ & $52(5)$ \\
Left atrial diameter (mm) & $39(7)$ & $42(6)$ \\
Ejection fraction (\%) & $67(8)$ & $64(10)$ \\
LV isovolumic & $121(21)$ & $124(23)$ \\
relaxation time (ms) & & \\
E/A ratio & $0.96(0.28)$ & $0.95(0.34)$ \\
\hline
\end{tabular}

PIVUS, Prospective Investigation of the Vasculature in Uppsala Seniors; ULSAM, Uppsala Longitudinal Study of Adult Men; BMI, body mass index; $\mathrm{HDL}$, high-density lipoprotein; $\mathrm{Cl}$, confidence interval; LV, left ventricular. ${ }^{a}$ Results are expressed as means (S.D.) or proportions. 
Table 2 Adiponectin in relation to echocardiographic measures in the PIVUS study $(n=954)^{\text {a }}$.

Associations with serum adiponectin

\begin{tabular}{|c|c|c|c|c|}
\hline & \multirow{2}{*}{\multicolumn{2}{|c|}{ Age- and sex-adjusted }} & \multirow{2}{*}{\multicolumn{2}{|c|}{ Multivariable-adjusted }} \\
\hline & & & & \\
\hline & $\beta(95 \% \mathrm{Cl})$ & $P$ value & $\beta(95 \% \mathrm{Cl})$ & $P$ value \\
\hline LV mass index $\left(\mathrm{g} / \mathrm{m}^{2.7}\right) ; n=895$ & $-1.48(-2.34 ;-0.62)$ & 0.001 & $0.42(-0.33 ; 1.17)$ & 0.28 \\
\hline LV relative wall thickness; $n=895$ & $-0.01(-0.01 ;-0.00)$ & 0.012 & $-0.00(-0.01 ; 0.00)$ & 0.27 \\
\hline LV end-diastolic diameter (mm); $n=897$ & $-0.21(-0.54 ; 0.12)$ & 0.21 & $0.32(-0.03 ; 0.67)$ & 0.073 \\
\hline Left atrial diameter $(\mathrm{mm}) ; n=924$ & $-0.70(-1.10 ;-0.29)$ & 0.001 & $0.25(-0.13 ; 0.63)$ & 0.20 \\
\hline Ejection fraction $(\%) ; n=810$ & $-0.73(-1.25 ;-0.20)$ & 0.007 & $-1.16(-1.73 ;-0.58)$ & $<0.0001$ \\
\hline $\mathrm{LV}$ isovolumic relaxation time $(\mathrm{ms}) ; n=871$ & $-0.75(-2.13 ; 0.64)$ & 0.29 & $0.79(-0.57 ; 2.16)$ & 0.25 \\
\hline $\log \mathrm{E} / \mathrm{A}$ ratio; $n=913$ & $0.02(-0.00 ; 0.03)$ & 0.070 & $0.00(-0.01 ; 0.02)$ & 0.60 \\
\hline
\end{tabular}

PIVUS, Prospective Investigation of the Vasculature in Uppsala Seniors; ULSAM, Uppsala Longitudinal Study of Adult Men; BMI, body mass index; HDL, highdensity lipoprotein: $\mathrm{Cl}$, confidence interval; LV, left ventricular.

${ }^{a}$ Values are $\beta$-coefficients ( $95 \%$ confidence intervals) from models with adiponectin together with age and sex (age-and sex-adjusted models), or with age, sex, $\mathrm{BMI}$, systolic blood pressure, antihypertensive medication, antidiabetic medication, lipid-lowering medication, log fasting glucose, total cholesterol, HDL cholesterol, creatinine, and smoking (multivariable-adjusted models) as independent variables, and echocardiographic measures as dependent variables in separate models. $\beta$-coefficients represent change in echocardiographic measure (in non-standardized units) per 1-s.D. increase in adiponectin.

interaction $=0.063)$; the association was evident in men $(\beta,-1.62 ; 95 \%$ confidence interval $(\mathrm{CI}),-2.50$, $-0.75 ; P<0.0001)$, but not in women $(\beta,-0.27 ; 95 \%$ CI, $-1.02,0.47 ; P=0.47)$. None of the other interaction terms were significant. The inverse relation between adiponectin and ejection fraction remained similar when excluding individuals with known prior CVD $(\beta,-0.70 ; 95 \%$ CI, $-1.29,-0.12 ; P=0.019$; $\beta,-1.62 ; 95 \% \mathrm{CI},-2.70,-0.55 ; P=0.003$, in PIVUS and ULSAM respectively). Exploratory analyses revealed that HDL cholesterol and BMI acted as negative confounders, i.e. they increased the magnitude of the association between adiponectin and ejection fraction when added to the age-and sex-adjusted model. LVM had no effect on the association between adiponectin and ejection fraction when added to the multivariable model, nor did further adjustment for C-reactive protein or homeostasis model assessment-IR (data not shown).

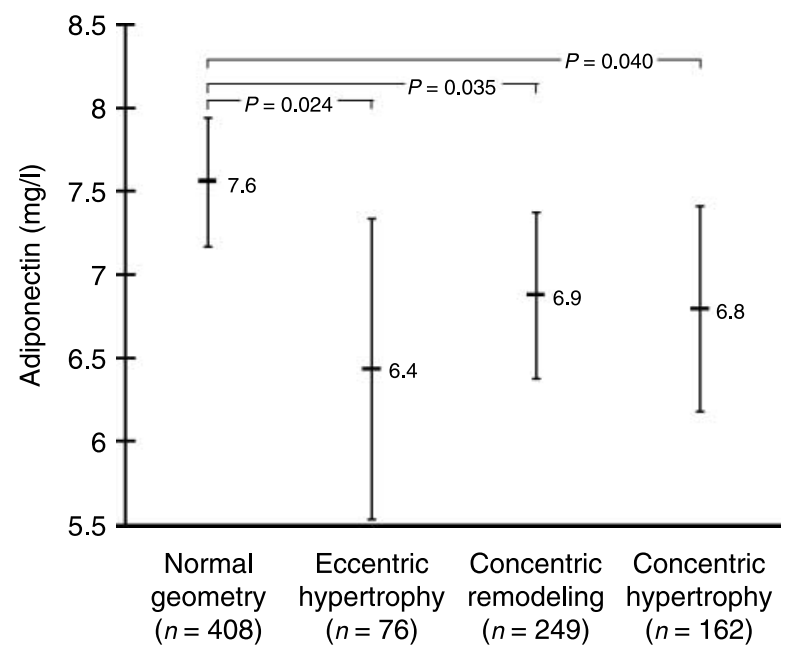

Figure 1 Age- and sex-adjusted least square means of adiponectin grouped by categories of cardiac geometry in the PIVUS study $(n=895)$.
To address the role of natriuretic peptides in mediating the association of adiponectin with cardiac function, we added NT-proBNP to the multivariableadjusted model with ejection fraction as an outcome. The association between adiponectin and ejection fraction was attenuated in both PIVUS $(\beta,-0.68$; $95 \%$ CI, $-1.25,-0.10 ; P=0.021)$ and ULSAM $(\beta,-0.75 ; 95 \% \mathrm{CI},-1.84,0.35 ; P=0.18)$. Consistent with the above sex-stratified analyses, the association between adiponectin and ejection fraction was attenuated, but still significant $(\beta,-0.98 ; 95 \%$ CI, -1.86 , $-0.10 ; P=0.029)$ in men and nonsignificant in women $(\beta,-0.14 ; 95 \% \mathrm{CI},-0.88,0.60 ; P=0.71)$ in PIVUS. Furthermore, the attenuation was lesser in individuals without prior CVD $(\beta,-0.69$ and -0.61 ; $95 \%$ CI, $-1.28,-0.11$ and -1.20 , -0.03 , without and with adjustment for NT-proBNP respectively), but more pronounced in a sub-sample with individuals having prior CVD $(\beta,-2.30$ and $-1.00 ; 95 \%$ CI, $-3.90,-0.69$ and $-2.76,-0.75$, without and with adjustment for NT-proBNP respectively).

With our sample sizes, we had at least $85 \%$ (PIVUS) or $81 \%$ (ULSAM) statistical power to detect an increment to the model $R^{2}$ of 0.01 (PIVUS) or 0.02 (ULSAM) for all measures of cardiac function and geometry (at $\alpha=0.05$ ).

\section{Discussion}

Our principal findings are twofold. First, adiponectin was inversely associated with ejection fraction in elderly men. This association was independent of potential confounders including BMI, and it was also evident in a sub-sample after exclusion of individuals with any previously known CVD. We did not observe an association between adiponectin and ejection fraction in women. Secondly, our results indicate that NT-proBNP may act as a partial mediator of the association of adiponectin with ejection fraction as the 
Table 3 Adiponectin in relation to echocardiographic measures in ULSAM $(n=427)^{\mathrm{a}}$.

\begin{tabular}{|c|c|c|c|c|}
\hline & \multicolumn{4}{|c|}{ Associations with serum adiponectin } \\
\hline & \multicolumn{2}{|c|}{ Age-adjusted } & \multicolumn{2}{|c|}{ Multivariable-adjusted } \\
\hline & $\beta(95 \% \mathrm{Cl})$ & $P$ value & $\beta(95 \% \mathrm{Cl})$ & $P$ value \\
\hline LV mass index $\left(\mathrm{g} / \mathrm{m}^{2.7}\right) ; n=427$ & $-0.26(-1.50 ; 0.98)$ & 0.69 & $1.08(-0.20 ; 2.36)$ & 0.10 \\
\hline LV relative wall thickness $(\mathrm{mm}) ; n=427$ & $-0.00(-0.01 ; 0.00)$ & 0.26 & $-0.00(-0.01 ; 0.01)$ & 0.66 \\
\hline LV end-diastolic diameter (mm); $n=427$ & $0.09(-0.41 ; 0.59)$ & 0.73 & $0.32(-0.24 ; 0.88)$ & 0.26 \\
\hline Left atrial diameter $(\mathrm{mm}) ; n=423$ & $-0.31(-0.86 ; 0.24)$ & 0.27 & $0.34(-0.26 ; 0.94)$ & 0.27 \\
\hline Ejection fraction (\%); $n=385$ & $-0.91(-1.86 ; 0.04)$ & 0.059 & $-1.35(-2.41 ;-0.29)$ & 0.013 \\
\hline LV isovolumic relaxation time $(\mathrm{ms}) ; n=378$ & $0.89(-1.39 ; 3.18)$ & 0.44 & $1.69(-0.89 ; 4.27)$ & 0.20 \\
\hline $\log \mathrm{E} / \mathrm{A}$ ratio; $n=387$ & $0.01(-0.02 ; 0.04)$ & 0.45 & $0.01(-0.02 ; 0.04)$ & 0.56 \\
\hline
\end{tabular}

ULSAM, Uppsala Longitudinal Study of Adult Men; BMI, body mass index; HDL, high-density lipoprotein; Cl, confidence interval; LV, left ventricular.

${ }^{a}$ Values are $\beta$-coefficients ( $95 \%$ confidence intervals) from models with adiponectin together with age (age-adjusted models), or with age, BMI, systolic blood pressure, antihypertensive medication, antidiabetic medication, lipid-lowering medication, log fasting glucose, total cholesterol, HDL cholesterol, creatinine, and smoking (multivariable-adjusted models) as independent variables, and echocardiographic measures as dependent variables in separate models. $\beta$-coefficients represent change in echocardiographic measure (in non-standardized units) per 1-s.D. increase in adiponectin.

$\beta$-coefficients of associations between adiponectin and ejection fraction were $40-50 \%$ lower when NT-proBNP was included in the models. Even if distinct conclusions cannot be drawn from cross-sectional studies, this apparent attenuation of effect may indicate that the associations of adiponectin and natriuretic peptides with LV systolic function could stem from the same pathophysiological pathways. The validity of our novel findings are underscored by the consistent findings in two large, independent community-based samples.

In recent years, the role of adiponectin has been of interest in several physiological processes, but there are only a few small studies trying to elucidate its relation with cardiac structure and function. Some prior studies have reported an inverse association between adiponectin and cardiac geometry, primarily LVM (5-9), but also ECG-LVH (10), whereas we observed such associations only in age- and sex-adjusted analyses, but not after adjustment for BMI. Consistent with this, adiponectin levels differed significantly between individuals with normal geometry and individuals with categories of hypertrophy and/or remodeling, adjusted for age and sex (Fig. 1). However, further adjustment for BMI rendered this association not significant, which highlights the role of BMI as a confounder of the association between adiponectin and cardiac geometry. Furthermore, in a study including 186 hypertensive patients and 89 healthy controls, high adiponectin levels associated inversely with $\mathrm{LV}$ isovolumic relaxation time and positively with $\mathrm{E} / \mathrm{A}$ ratio (7), i.e. with less diastolic dysfunction. However, these studies have been performed in small samples of younger patients, often with preexisting disease, such as type 2 diabetes (8), hypertenstion (7), or obesity $(5,6)$, and confounders have not always been adjusted for (8). These differences in the study design are plausible explanations for the discrepancies.

Circulating adiponectin levels are higher in patients with prevalent heart failure (23), and they have been shown to correlate with circulating NT-proBNP in patients with heart failure (12-14). Also, in heart failure patients, adiponectin has been demonstrated to be a prognosticator of mortality and hospitalizations for heart failure $(12,23)$. Recently, we reported that neither circulating adiponectin nor genetic variation in the adiponectin gene (ADIPOQ) predicted incident heart failure in the ULSAM, where we followed 946 men without heart failure for up to 11 years (24). This was corroborated by a recent report from the Framingham Heart Study (25), where no associations of adiponectin with incident heart failure were found, whereas circulating resistin was associated with incident heart failure, even when taking prevalent coronary heart disease, obesity, and measures of IR and inflammation into account. In a recent study of 608 healthy individuals from Japan in an age group that was comparable to that of the present study (mean age, 62 years), adiponectin was increased with higher LVEDD even after adjustment for age, sex, and BMI (11). Furthermore, adiponectin tracked with BNP; thus, the authors suggested that adiponectin might reflect some aspects of cardiac function. This was supported by a recent case-control study of 250 CVD patients and 250 controls where circulating adiponectin levels were positively associated with NT-proBNP, especially in the higher range of NT-proBNP levels (26). Taken together, these observations clearly substantiate the findings of the present study.

To put the decrease of $1.2 \%$ in ejection fraction per 1 -s.D. increase of adiponectin in perspective and understand its potential clinical relevance, we compared this effect size with the other two significant continuous predictors of ejection fraction in our multivariableadjusted model (change in ejection fraction, -0.93 and $1.00 \%$ per 1-S.D. increase of BMI and systolic blood pressure respectively). Nevertheless, even if adiponectin demonstrated the highest effect size among the clinical predictors, the question of how large ejection fraction change is clinically relevant is not trivial, since it depends on other factors, such as age, sex, and 
concomitant congestive heart failure, and where in the distribution of ejection fraction this change occurs (as there is a nonlinear relation of ejection fraction with outcome). However, as an example, a 5\% increment in ejection fraction was associated with a $13 \%$ lower hazard of death in individuals with heart failure in the Framingham Heart Study (27). In any case, it is important to emphasize that the main objective of the present study was to disentangle mechanisms linking adiponectin with cardiac geometry and function rather than finding clinically useful predictors of LV function.

In the present study, we observed associations of adiponectin with ejection fraction in men, but not in women. This could possibly be explained by previously demonstrated sex differences in circulating adiponectin levels in human samples, with females having higher levels than males $(28,29)$. There are some indications that this sex difference in circulating levels of adiponectin could be explained by an inhibitory influence of androgens. One study demonstrated significantly increased levels of adiponectin in castrated male mice, whereas injections of testosterone in these mice decreased plasma adiponectin (30). Analysis in cultured adipocytes showed that testosterone and $5 \alpha$-DHT suppressed the secretion of adiponectin. Since adiponectin mRNA remained unaffected by testosterone treatment in vitro and in vivo, the existence of a testosterone-regulated factor involved in the secretion of adiponectin was suggested (30). Consistent with this, a small study in normal men reported significantly increased concentrations of adiponectin upon experimental reduction of testosterone and decreased levels after the administration of testosterone (31).

The notion that adiponectin is associated with prevalent, but not incident heart failure, has spurred the idea that the cardiac cachexia seen in many heart failure patients could lead to lower fat mass and consequently to higher adiponectin levels. However, the present study was performed in healthy, free-living individuals without overt disease, and the inverse association between adiponectin and systolic function was also present in a healthier sub-sample (after exclusion of all individuals with any prior CVD) and after adjustments for BMI along with other potential confounders. These observations, along with the prior study of similarly healthy elderly individuals from Japan (11), suggest that cardiac cachexia is unlikely to be the sole reason for the associations of circulating adiponectin, NT-proBNP, and LV systolic function. Nevertheless, it should be noted that the attenuation of the association of adiponectin with ejection fraction was even more pronounced in a sub-sample of individuals with prior CVD. However, this observation needs to be confirmed in other settings as the sample size for these secondary analyses was too modest to draw firm conclusions.

Given the previously reported associations between increased adiponectin and BNP concentrations along with heart failure (12-14), or LV dilation in individuals without overt diease (11), we hypothesized that adiponectin would be involved in the same cardioregulatory pathway as BNP. Our analyses showed that adjustment for NT-proBNP partially attenuated the association between adiponectin and ejection fraction. A potential explanation could be that BNP is along the pathway by which adiponectin is associated with LV systolic function. Previous studies have shown that natriuretic peptides exhibit a lipolytic effect on human adipose tissue through a cGMP-dependent pathway (32-34) that may explain the linkage between adiponectin and BNP. Circulating adiponectin has been suggested to be a marker of total stimulated adipose tissue triacylglycerol lipolytic capacity in vivo in humans (35). Another study demonstrated that infusion of synthetic atrial natriuretic peptide (ANP) increased levels of total and high-molecular weight adiponectin in patients with heart failure (36). In addition, a preliminary study has shown that both ANP and BNP increased the adiponectin mRNA expression in a dosedependent manner, which was mediated through the cGMP-dependent pathway (37).

Strengths of the present study include the confirmation of the study results in two large, independent community-based samples with comprehensive assessment of cardiac geometry and function. However, some limitations should be acknowledged. First, since both study samples consisted exclusively of elderly individuals of Northern European ethnicity, it is unknown if the results can be generalized to younger individuals or other ethnicities. Secondly, measures of different multimeric forms of serum adiponectin were not available for the present study. This could be of some importance since some studies suggest a difference in biological activity between different isoforms of adiponectin with regard to metabolic abnormalities $(38,39)$. Thirdly, as this is a cross-sectional study, causality cannot be elucidated nor can it be determined if cardiac geometry and function are related to the longitudinal tracking of adiponectin concentrations. Fourthly, ejection fraction was measured according to the Teichholz formula using M-mode in both cohorts. This way of evaluating ejection fraction has its known limitations since it does not take into consideration dyskinesia in segments other than those included in the M-mode recording. Despite this limitation, this way of measuring ejection fraction is still used very frequently in the clinical setting, with a substantial part of the literature regarding the predictive power of ejection fraction, and inclusion criteria in landmark heart failure treatment trials are based on this method. Lastly, the samples were stored at $-70{ }^{\circ} \mathrm{C}$ for 1-3 years (PIVUS) and $11 \pm 2$ years (ULSAM) before being analyzed. However, at the time of collection, the samples were ideally treated to limit any influence on stability of samples. The effect of frozen storage on adiponectin levels has to our best knowledge been investigated under a maximum of 30 months, with no discernible effect of mean plasma adiponectin 
levels being reported (40). We are not aware of any other studies with longer follow-up, but most peptide hormones have been shown to be fairly stable, some peptides even during long-term storage up to 25 years $(41)$.

In conclusion, in our two community-based samples of the elderly, adiponectin concentrations were inversely associated with ejection fraction in men, even after adjustment for potential confounders including BMI. These associations were partially attenuated by additional adjustment for NT-proBNP, indicating that adiponectin may be associated with depressed LV systolic function through pathways shared with natriuretic peptides. Further studies are required to confirm our findings, and to establish the biological pathways linking adiponectin, natriuretic peptides, and cardiac function.

\section{Declaration of interest}

The authors declare that there is no conflict of interest that could be perceived as prejudicing the impartiality of the research reported.

\section{Funding}

Sources of support: This work was supported by the Swedish Research Council (grant number 2007-2135), Swedish Heart-Lung Foundation (grant number 20070212), Royal Swedish Academy of Science, Linnéus Foundation for Medical Research, the Foundation of Erik, Karin och Gösta Selander, the Foundation of Fredrik and Ingrid Thuring, the Foundation of Åke Wiberg, the Northern Sweden Councils (Visare Norr), the Swedish Society for Medical Research, AstraZeneca R\&D Mölndal and the Danish Medical Research Council.

Role of the sponsor: The sponsors had no role in the study design, analyses, writing, or decision to publish the manuscript.

\section{References}

1 Guilbert JJ. The world health report 2002 - reducing risks, promoting healthy life. Education for Health 200316230

2 Prevention of Cardiovascular Disease: Guideline for Assessment and Management of Cardiovascular Risk. Geneva: World Health Organization, 2007.

3 Matsuzawa Y. Therapy insight: adipocytokines in metabolic syndrome and related cardiovascular disease. Nature Clinical Practice. Cardiovascular Medicine 20063 35-42.

4 Nishida M, Funahashi T \& Shimomura I. Pathophysiological significance of adiponectin. Medical Molecular Morphology 2007 40 55-67.

5 Ebinc H, Ebinc FA, Ozkurt ZN, Dogru MT, Tulmac M, Yilmaz M \& Caglayan O. Impact of adiponectin on left ventricular mass index in non-complicated obese subjects. Endocrine Journal $2008 \mathbf{5 5}$ 523-528.

6 Ybarra J, Pou JM, Planas F, Ballesta-Lopez C, Echevarne F, Romeo JH \& Navarro-Lopez F. Correlation between insulin resistance surrogates and echocardiographic findings in asymptomatic patients with morbid obesity: a cross-sectional study. Endocrine Practice 200713 590-600.

7 Hong SJ, Park CG, Seo HS, Oh DJ \& Ro YM. Associations among plasma adiponectin, hypertension, left ventricular diastolic function and left ventricular mass index. Blood Pressure 200413 236-242.

8 Top C, Sahan B \& Onde ME. The relationship between left ventricular mass index and insulin sensitivity, postprandial glycaemia, and fasting serum triglyceride and adiponection levels in patients with type 2 diabetes. Journal of International Medical Research 200735 909-916.

9 Kozakova M, Muscelli E, Flyvbjerg A, Frystyk J, Morizzo C, Palombo C \& Ferrannini E. Adiponectin and left ventricular structure and function in healthy adults. Journal of Clinical Endocrinology and Metabolism 200893 2811-2818.

10 Mitsuhashi H, Yatsuya H, Tamakoshi K, Matsushita K, Otsuka R, Wada K, Sugiura K, Takefuji S, Hotta Y, Kondo T, Murohara T \& Toyoshima H. Adiponectin level and left ventricular hypertrophy in Japanese men. Hypertension 200749 1448-1454.

11 Ohara T, Kim J, Asakura M, Asanuma H, Nakatani S, Hashimura K, Kanzaki H, Funahashi T, Tomoike H \& Kitakaze M. Plasma adiponectin is associated with plasma brain natriuretic peptide and cardiac function in healthy subjects. Hypertension Research 200831 825-831.

12 Kistorp C, Faber J, Galatius S, Gustafsson F, Frystyk J, Flyvbjerg A \& Hildebrandt P. Plasma adiponectin, body mass index, and mortality in patients with chronic heart failure. Circulation 2005 112 1756-1762.

13 Haugen E, Furukawa Y, Isic A \& Fu M. Increased adiponectin level in parallel with increased NT-pro BNP in patients with severe heart failure in the elderly: a hospital cohort study. International Journal of Cardiology 2008125 216-219.

14 Tamura T, Furukawa Y, Taniguchi R, Sato Y, Ono K, Horiuchi H, Nakagawa Y, Kita T \& Kimura T. Serum adiponectin level as an independent predictor of mortality in patients with congestive heart failure. Circulation Journal 200771 623-630.

15 Lind L, Fors N, Hall J, Marttala K \& Stenborg A. A comparison of three different methods to evaluate endothelium-dependent vasodilation in the elderly: the Prospective Investigation of the Vasculature in Uppsala Seniors (PIVUS) study. Arteriosclerosis, Thrombosis, and Vascular Biology 200525 2368-2375.

16 Hedstrand H. A study of middle-aged men with particular reference to risk factors for cardiovascular disease. Upsala Journal of Medical Sciences. Supplement 197519 1-61.

17 Sundström J, Ärnlöv J, Stolare K \& Lind L. Blood pressureindependent relations of left ventricular geometry to the metabolic syndrome and insulin resistance: a population-based study. Heart $200894874-878$.

18 Devereux RB, Alonso DR, Lutas EM, Gottlieb GJ, Campo E, Sachs I \& Reichek N. Echocardiographic assessment of left ventricular hypertrophy: comparison to necropsy findings. American Journal of Cardiology $1986 \mathbf{5 7} 450-458$.

19 de Simone G, Daniels SR, Devereux RB, Meyer RA, Roman MJ, de Divitiis O \& Alderman MH. Left ventricular mass and body size in normotensive children and adults: assessment of allometric relations and impact of overweight. Journal of the American College of Cardiology 199220 1251-1260.

20 Teichholz LE, Kreulen T, Herman MV \& Gorlin R. Problems in echocardiographic volume determinations: echocardiographicangiographic correlations in the presence of absence of asynergy. American Journal of Cardiology 197637 7-11.

21 Ganau A, Devereux RB, Roman MJ, de Simone G, Pickering TG, Saba PS, Vargiu P, Simongini I \& Laragh JH. Patterns of left ventricular hypertrophy and geometric remodeling in essential hypertension. Journal of the American College of Cardiology 199219 1550-1558.

22 Frystyk J, Berne C, Berglund L, Jensevik K, Flyvbjerg A \& Zethelius B. Serum adiponectin is a predictor of coronary heart disease: a population-based 10-year follow-up study in elderly men. Journal of Clinical Endocrinology and Metabolism 200792 571-576.

23 George J, Patal S, Wexler D, Sharabi Y, Peleg E, Kamari Y, Grossman E, Sheps D, Keren G \& Roth A. Circulating adiponectin concentrations in patients with congestive heart failure. Heart 200692 1420-1424.

24 Ingelsson E, Riserus U, Berne C, Frystyk J, Flyvbjerg A, Axelsson T, Lundmark P \& Zethelius B. Adiponectin and risk of congestive heart failure. Journal of the American Medical Association 2006 295 1772-1774. 
25 Frankel DS, Vasan RS, D’Agostino RB Sr, Benjamin EJ, Levy D, Wang TJ \& Meigs JB. Resistin, adiponectin, and risk of heart failure the Framingham offspring study. Journal of the American College of Cardiology 200953 754-762.

26 Soderberg S, Colquhoun D, Keech A, Yallop J, Barnes EH, Pollicino C, Simes J, Tonkin AM \& Nestel P. Leptin, but not adiponectin, is a predictor of recurrent cardiovascular events in men: results from the LIPID study. International Journal of Obesity 200933 123-130.

27 Vasan RS, Larson MG, Benjamin EJ, Evans JC, Reiss CK \& Levy D. Congestive heart failure in subjects with normal versus reduced left ventricular ejection fraction: prevalence and mortality in a population-based cohort. Journal of the American College of Cardiology 199933 1948-1955.

28 Cnop M, Havel PJ, Utzschneider KM, Carr DB, Sinha MK, Boyko EJ, Retzlaff BM, Knopp RH, Brunzell JD \& Kahn SE. Relationship of adiponectin to body fat distribution, insulin sensitivity and plasma lipoproteins: evidence for independent roles of age and sex. Diabetologia $2003 \mathbf{4 6} 459-469$.

29 Yamamoto Y, Hirose H, Saito I, Tomita M, Taniyama M, Matsubara K, Okazaki Y, Ishii T, Nishikai K \& Saruta T. Correlation of the adipocyte-derived protein adiponectin with insulin resistance index and serum high-density lipoprotein-cholesterol, independent of body mass index, in the Japanese population. Clinical Science $2002 \mathbf{1 0 3} 137-142$.

30 Nishizawa H, Shimomura I, Kishida K, Maeda N, Kuriyama H, Nagaretani H, Matsuda M, Kondo H, Furuyama N, Kihara S, Nakamura T, Tochino Y, Funahashi T \& Matsuzawa Y. Androgens decrease plasma adiponectin, an insulin-sensitizing adipocytederived protein. Diabetes 200251 2734-2741.

31 Page ST, Herbst KL, Amory JK, Coviello AD, Anawalt BD, Matsumoto AM \& Bremner WJ. Testosterone administration suppresses adiponectin levels in men. Journal of Andrology 2005 26 85-92.

32 Sengenes C, Berlan M, De Glisezinski I, Lafontan M \& Galitzky J. Natriuretic peptides: a new lipolytic pathway in human adipocytes. FASEB Journal 200014 1345-1351.

33 Sengenes C, Bouloumie A, Hauner H, Berlan M, Busse R, Lafontan M \& Galitzky J. Involvement of a cGMP-dependent pathway in the natriuretic peptide-mediated hormone-sensitive lipase phosphorylation in human adipocytes. Journal of Biological Chemistry 2003278 48617-48626.
34 Moro C, Crampes F, Sengenes C, De Glisezinski I, Galitzky J, Thalamas C, Lafontan M \& Berlan M. Atrial natriuretic peptide contributes to physiological control of lipid mobilization in humans. FASEB Journal 200418 908-910.

35 Lavoie F, Frisch F, Brassard P, Normand-Lauziere F, Cyr D, Gagnon R, Drouin R, Baillargeon JP \& Carpentier AC. Relationship between total and high molecular weight adiponectin levels and plasma nonesterified fatty acid tolerance during enhanced intravascular triacylglycerol lipolysis in men. Journal of Clinical Endocrinology and Metabolism 2009 94 998-1004.

36 Tanaka T, Tsutamoto T, Sakai H, Nishiyama K, Fujii M, Yamamoto T \& Horie M. Effect of atrial natriuretic peptide on adiponectin in patients with heart failure. European Journal of Heart Failure 200810 360-366.

37 Tsukamoto O, Fujita M, Kato M, Yamazaki S, Asano Y, Ogai A, Okazaki H, Asai M, Nagamachi Y, Maeda N, Shintani Y, Minamino T, Asakura M, Kishimoto I, Funahashi T, Tomoike H \& Kitakaze M. Natriuretic peptides enhance the production of adiponectin in human adipocytes and in patients with chronic heart failure. Journal of the American College of Cardiology 200953 2070-2077.

38 Hara K, Horikoshi M, Yamauchi T, Yago H, Miyazaki O, Ebinuma H, Imai Y, Nagai R \& Kadowaki T. Measurement of the high-molecular weight form of adiponectin in plasma is useful for the prediction of insulin resistance and metabolic syndrome. Diabetes Care 200629 1357-1362.

39 Pajvani UB, Hawkins M, Combs TP, Rajala MW, Doebber T, Berger JP, Wagner JA, Wu M, Knopps A, Xiang AH, Utzschneider KM, Kahn SE, Olefsky JM, Buchanan TA \& Scherer PE. Complex distribution, not absolute amount of adiponectin, correlates with thiazolidinedione-mediated improvement in insulin sensitivity. Journal of Biological Chemistry 2004 $27912152-12162$.

40 Shand B, Elder P, Scott R, Frampton C \& Willis J. Biovariability of plasma adiponectin. Clinical Chemistry and Laboratory Medicine 200644 1264-1268.

41 Zethelius B, Byberg L, Hales CN, Lithell H \& Berne C. Proinsulin is an independent predictor of coronary heart disease: report from a 27-year follow-up study. Circulation 2002105 2153-2158.

Received 21 November 2009

Accepted 11 December 2009 